\title{
MOLECULAR DYNAMICS SIMULATION MODEL OF AFM-BASED NANOMACHINING
}

\author{
Rapeepan Promyoo ${ }^{1,3}$, Hazim El-Mounayri ${ }^{1,3, *}$ and Kody Varahramyan ${ }^{2,3}$ \\ ${ }^{1}$ Department of Mechanical Engineering, Indiana University Purdue University \\ Indianapolis, Indianapolis, IN, USA \\ ${ }^{2}$ Department of Electrical and Computer Engineering, Indiana University \\ Purdue University Indianapolis, Indianapolis, IN, USA \\ ${ }^{3}$ Integrated Nanosystems Development Institute (INDI), \\ IUPUI, Indianapolis, IN, USA \\ *Corresponding author (helmouna@iupui . edu)
}

\begin{abstract}
In this paper, a developed three-dimensional Molecular Dynamics (MD) model for AFM-based nanomachining is applied to study mechanical indentation and scratching at the nanoscale. The correlation between the machining conditions, including applied force, depth, tip speed, and defect mechanism in substrate/workpiece is investigeted. The simulations of nanoscratching process are performed on different crystal orientations of single-crystal gold substrate, $A u(100)$, $A u(110)$, and $A u(111)$. The material deformation and deformed geometry are extracted from the final locations of atoms, which are displaced by the rigid indenter. The simulation also allows for the prediction of forces at the interface between the indenter and substrate. Material properties including modulus of elasticity and hardness are estimated. It is found that properties vary significantly at the nanoscale. In addition to the modeling, an AFM is used to conduct actual indentation and scratching at the nanoscale, and provide measurements to which the MD simulation predictions are compared. Due to computational time limitation, the predicted forces obtained from MD simulation only compares well qualitatively with the experimental results.
\end{abstract}

\section{KEYWORDS}

AFM-based Nanomachining, Molecular Dynamics (MD), Nanoindentation, Nanoscratching, Simulation

\section{INTRODUCTION}

Atomic force microscope (AFM) has been considered a potential manufacturing tool for operations including machining, patterning, and assembling with in situ metrology and visualization [1]. AFM-based nanomachining generally involves nanoindentation and nanoscratching, which have been commonly used in the characterization of surfaces or smallscale materials [2]. It also has the ability to perform in situ repair/re-manufacturing of the position, size, shape, and orientation of single nanostructures. Some applications of AFM-based nanomachining include fabrication of micro-/nano-devices, individualized biomedicine and drug delivery, molecular reading and sorting, ultrahigh density memory, nanoscale circuitry, and fabrication of metal nanowires [3-13].

David C. Wyld et al. (Eds) : SAI, CDKP, ICAITA, NeCoM, SEAS, CMCA, ASUC, Signal - 2014 pp. 151-168, 2014. (C) CS \& IT-CSCP 2014

DOI : $10.5121 /$ csit.2014.41115 
Recently, AFM tips have been used as cutting tools for surface modification. Nanochannels, nanoslots, and complex nanopatterns can be fabricated by directly scratching the substrate [9]. These AFM-based mechanical indentation and scratching techniques have been successfully applied to produce complex geometries and high aspect-ratio 3D nano-objects on both flat and curved surfaces [10]. Nanoindentation and nanoscratching are capable of fabricating complex structures, and advances in materials, pattern transfer processes, and cost reductions of AFM equipment have allowed these methods to become a viable but not yet scalable method for many nanoscale devices [14]. Process throughput is low due to limited removal speed, tip-surface approach, contact detection, desired force profile, and tool wear. Parallel fabrication using multiple AFM tip arrays has been reported [5]. However, parallel fabrication currently does not allow precise control over size, shape, position, or orientation of individual structures. A fundamental understanding of substrate deformations/separations and the tip is needed to achieve controllable nanomanufacturing [1]. Attempts have been made to study the correlation between machining parameters, machined geometry, and surface properties for better control of AFMbased nanomachining processes both experimentally [15-21] and computationally [22-55]. This include experiments on few types of materials to investigate the effects of parameters such as applied load, scratching speed, feed rate, scratching direction, tip geometry, tip angle, tip radius, and number of scratching cycles. These parameters which also depend on material properties and crystal orientation of the substrate, affect the depth, width, chip formation, and surface roughness of the machined surface. Due to experimental limitations, computational models are therefore essential to achieve a more comprehensive/complete understanding of the roles of the parameters affecting the final nano-geometry in AFM-based nanomachining. On the other hand, a more extensive experimental study is necessary to inform the development of accurate and realistic predictive models. The experimental data is also needed to validate the computational models.

To address the need for computational models of AFM-based nanomachining, some efforts have been made to model nanoindentation and nanoscratching using MD simulations [22-55]. MD simulation presents itself as a viable alternative to the expensive traditional experimental approach. Such a simulation was initiated in the late 1950s by Alder and Wainwright [56-57] in the field of statistical mechanics and has been successfully applied to investigate various phenomena at nanoscale. The advantage of MD simulation over continuum model simulation (FE) is that it allows for a better, more detailed understanding of the ways defects are created, the transition from elastic to plastic behavior, and crystal structure effects in materials [22]. Numerous studies have been reported on MD simulations of nanoindentaion and nanoscratching. The effects of several parameters such as crystal orientation [41, 45, 46], indenter shape and orientation [33, 39, 40, 44], penetration or scratching depth [37, 42, 47, 48], scratching speed [47, 48], feed (on nanoscratching) [34, 35], and temperature [25, 45, 49] have been investigated on different types of bulk and thin film materials. In addition, mechanical properties including Young's modulus, friction coefficient and hardness of materials have also been reported [26, 42]. MD simulation quality depends on the accuracy of the potential energy function used. Also, the complexity of the potential energy function directly affects computational time. The selection of the potential function depends on material type. Various types were investigated in MD simulations: silicon [22, 30, 31], gold [32], copper [25, 33-35], aluminum [36-38], silver [39, 40], iron [41, 42] and nickel [43, 44]. However, MD simulation involves the interaction of a large number of atoms as deformation occurs on an atomic scale. One major concern in MD simulation is the high computational time required. Existing MD models are limited in the size of simulated volume as well as time scale, inhibiting the ability to capture all important attributes for deformation. To keep the processing time under control, most existing models of nanoindentation use less than 100,000 atoms. The largest models of nanoindentation found in the literature contain approximately 10 million atoms [58], which are enabled by parallel computing.

In this paper, three-dimensional MD simulations of AFM nanoindentation and nanoscratching are performed to investigate the effects of tip speed and crystal orientation for the case of gold material. The simulation allows for the prediction of forces at the interface between an indenter 
and a substrate. The material deformation and deformed geometry are extracted based on the final locations of the atoms, which have been displaced by the rigid tool. Mechanical properties including Young's modulus and hardness of materials are also reported. In addition, an AFM is used to conduct actual indentation and scratching at the nanoscale, and provide data with which to validate MD simulation. The results of the simulation as well as the AFM data are presented and compared.

\section{METHODOLOGY}

MD simulation is used to simulate the time dependent behavior of a molecular system. MD simulations of AFM-based nanomachining in this study are implemented using LAMMPS (Large-scale Atomic/Molecular Massively Parallel Simulator) [59, 60]. The LAMMPS code run in parallel uses distributed memory message passing techniques and spatial decomposition of simulation domain. The inputs required in MD simulation are initial positions and velocities of atoms in the system along with other information such as boundary conditions, potential energy function, time steps, etc. The outputs of the simulation include trajectories of atoms in the system, forces, energy of the system, and other physical quantities of interest. The MD simulation model and the potential functions used in this study are explained in the following sections.

\subsection{Simulation Model}

The schematic model used in the MD simulation of AFM nanoscratching is shown in Figure 1. The simulation model consists of a single crystal gold workpiece and a three-sided pyramidal indenter. Diamond is selected as indenter tip. The indenter tip is modeled as a rigid body. The initial positions of atoms in the model are calculated from the default lattice position. For example, face center cubic (fcc) structure is applied in the modeling of gold workpieces. On the other hand, diamond structure is used for modeling of diamond indenter. The workpiece in the MD simulation is divided into three different zones: boundary, thermostat, and the Newtonian zones. A few layers of boundary and thermostat atoms are placed on the bottom side of the workpiece. Fixed boundary conditions are applied to the boundary atoms. The atoms are fixed in the position to reduce the edge effects and maintain the symmetry of the lattice. Periodic boundary conditions are maintained along the $\mathrm{x}$ - and $\mathrm{y}$-direction. The periodic boundary conditions are usually employed when a simulation seek to investigate the behavior of an isolated system, to avoid spurious edge effects and thereby simulate the behavior of a much larger crystal system. The thermostat zone is applied to the MD simulation model to ensure that the heat generated during the indentation process can be conducted out of the indentation region properly. The temperature in the thermostat zone is maintained by scaling the velocities of the thermostat atoms for each computational time step. In the Newtonian zone, atoms move according to Newton's equation of motion. 


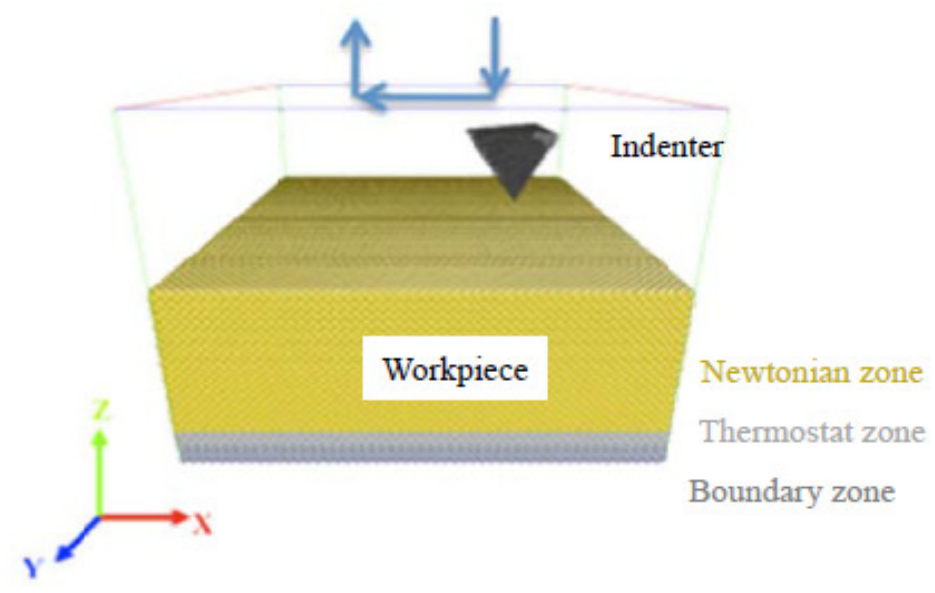

Figure 1. Schematic MD simulation model of AFM nanoscratching

\subsection{Potential Energy Function}

The motion of the atoms in the Newtonian zone is determined by the forces derived from potential energy function and Newton's equation of motion. The interaction of each atom can be approximated by a potential energy function in accordance with Newtonian mechanics. The quality of the MD simulation results depends on the accuracy of the potential energy function used. On the other hand, the complexity of the potential energy function directly affects the computational time [61]. The selection of the potential function depends on the type of material used in the model. The potential energy function used for the interaction between atoms in the gold $(\mathrm{Au})$ workpiece materials is the Embedded Atom Method (EAM) potential [62]. The Morse potential [63] is employed for the interaction between the gold workpiece and diamond indenter tip in the MD simulations.

The Morse potential [63] is a commonly used empirical potential energy function for bonded interactions The Morse potential energy function $U$ can be expresses as a function of interatomic distance $r$ as the following formula:

$$
U(r)=D\left\{\exp \left[-2 \alpha\left(r-r_{e}\right)\right]-2 \exp \left[-\alpha\left(r-r_{e}\right)\right]\right\}
$$

where $r$ is the distance between the atoms, $r_{e}$ is the equilibrium bond distance, $D$ is the cohesive energy, and $\alpha$ is a parameter controlling the width of the potential. The single independent variable in the equation is $r$. The constant parameters, $r_{e}, \alpha$, and $D$, can be determined on the basis of the physical properties of the material. The parameters used in the Morse potential for gold are listed in Table 1. The parameters between gold and carbon (Au-C) are calculated from the following equations.

$$
\begin{aligned}
& D_{A u-C}=\sqrt{D_{A u} \cdot D_{C}} \\
& \alpha_{A u-C}=\sqrt{\alpha_{A u} \cdot \alpha_{C}} \\
& r_{e A u-C}=\sqrt{r_{e A u} \cdot r_{e C}}
\end{aligned}
$$

The EAM potential [62] is an extension of the two-body potential that has been developed for metals. The basic approach of the EAM, which evolved from the density-function theory, is based upon the recognition that the cohesive energy of a metal is governed not only by the pair-wise 
potential of the nearest neighbor atoms, but also by embedding energy related to the electron gas that surrounds each atom.

Table 1. Parameters used in the Morse potential energy function

\begin{tabular}{|c|c|c|c|}
\hline Parameter & $\begin{array}{c}\mathrm{Au}-\mathrm{Au} \\
{[64]}\end{array}$ & $\begin{array}{c}\mathrm{C}-\mathrm{C} \\
{[25]}\end{array}$ & $\mathrm{Au}-\mathrm{C}$ \\
\hline$D(\mathrm{eV})$ & 0.475 & 2.423 & 1.073 \\
$\alpha\left(\AA^{-1}\right)$ & 1.583 & 2.555 & 2.011 \\
$r_{e}(\AA)$ & 3.024 & 2.522 & 2.762 \\
\hline
\end{tabular}

The interatomic force between any two atoms can be obtained from the potential energy function $(U)$ such that

$$
F_{i j}=-\frac{\partial U}{\partial r_{i j}}
$$

where $F_{i j}$ is the interatomic force between atom $i$ and $j$ at a distance $r_{i j}$ from atom $i$. The total force exerted on a particular atom is then calculated as the following equation.

$$
F_{i}=\sum_{j=1, i \neq j}^{N} F_{i j}\left(r_{i j}\right)
$$

where $F_{i}$ is the resultant force on atom $i$ and $N$ is the total number of atoms. After calculating force on each atom, velocities and positions are calculated from Newton's second law of motion.

In this study, material properties, Young's modulus and hardness, are calculated using the formulations developed by Oliver and Pharr [65]. They used data directly drawn from the loaddisplacement curve and correlated the projected contact area, $A_{c}$, to the contact depth, $h_{c}$, where $h_{c}$ may be expresses as

$$
h_{c}=h_{\max }-0.72 \frac{P_{\max }}{S_{\max }}
$$

where $h_{\max }$ is the maximum depth of indentation, $P_{\max }$ is the maximum applied load and $S_{\max }$ is the slope of the unloading curve at the maximum applied load. The contact area, $A_{c}$, is thus found from the geometry of the indenter as a function of the contact depth, $h_{c}$. Once the contact area is known, the hardness, $H$, is estimated from the maximum indentation load $P_{\max }$ divided by the projected contact area, i.e.

$$
H=\frac{P_{\max }}{A_{c}}
$$

The Young's modulus is calculated by the reduced elastic modulus, $E_{r}$, which takes into account the combined elastic effects of indenter tip and sample, as follows:

$$
E_{r}=\frac{1}{2} \sqrt{\frac{\pi}{A_{c}}} \frac{d P}{d h}
$$

where $d P / d h$ is the slope of tangent line at the beginning of the unloading curve and $A_{c}$ is the projected area at the maximum depth of indentation. The Young's modulus of the sample, $E_{s}$, is then calculated from the following equation. 


$$
\frac{1}{E_{r}}=\frac{1-v_{s}^{2}}{E_{s}}+\frac{1-v_{i}^{2}}{E_{i}}
$$

where $E_{i}$ is the Young's modulus of the indenter, and $v_{s}$ and $v_{i}$ are the Poisson's ratios of the sample and indenter, respectively.

\subsection{Ensembles of Statistical Thermodynamics}

Statistical ensembles are usually characterized by fixed values of thermodynamic variables such as energy, E, temperature, $\mathrm{T}$, pressure, $\mathrm{P}$, volume, $\mathrm{V}$, particle number, $\mathrm{N}$, or chemical potential, $\mu$. One fundamental ensemble is called the microcanonical ensemble and is characterized by constant particle number, $\mathrm{N}$, constant volume, $\mathrm{V}$ and constant total energy, $\mathrm{E}$, and is denoted as the NVE ensemble. Other examples include the canonical, or NVT ensemble, the isothermalisobaric or NPT ensemble, and the grand canonical or $\mu \mathrm{VT}$ ensemble. In the current study, microcanonical or NVE ensemble is applied in the Newtonian zone. The system is isolated from changes in number of atoms $(\mathrm{N})$, volume $(\mathrm{V})$ and energy $(\mathrm{E})$. It corresponds to an adiabatic process with no heat exchange. A microcanonical molecular dynamics trajectory may be seen as an exchange of potential and kinetic energy, with total energy being conserved.

\subsection{Parallel MD Simulation}

The parallel MD simulations of AFM-based nanomachining are implemented using LAMMPS $[59,60]$. The LAMMPS code run in parallel uses distributed memory message passing techniques and spatial decomposition of simulation domain. In spatial decomposition, the simulation domain is divided into a set of equal smaller sized domains. Each sub-domain is distributed to different processor for calculation. Since nearby atoms are placed on same processor, only neighboring atoms on different processor need to be communicated by Message Passing Interface (MPI). Communication is minimized to optimal level by replicating force computations of boundary atoms. Non-uniformity of data distribution can occur for spatial decomposition as interaction between tool and workpiece arise. The parallel MD simulation is run on the Big Red II supercomputer [66]. Big Red II is Indiana University's main system for high-performance parallel computing. Big Red II combines the longstanding leadership of Cray supercomputers with IUdeveloped technology. The Cray XE6/XK7 supercomputer is capable of one thousand trillion floating-point operations per second, or one petaFLOPS, making it the fastest university-owned supercomputer in the world.

\subsection{Simulation Conditions}

MD simulations of AFM-based nanomachining were conducted on single crystal gold with the use of parallel computing. Table 2 gives the conditions used in the MD simulations of AFMbased nanomachining. The dimensions of the workpiece and indenter, the depth of indentation and the tip speeds are given. The dimensions of the workpiece are expressed in terms of the lattice constants. The lattice constant of gold $(\mathrm{aAu})$ is 4.080 Angstroms $(\AA)$.

Table 2. MD simulation conditions used in the MD simulations of AFM-based nanomachining

\begin{tabular}{|l|l|}
\hline Workpiece material & Gold $(\mathrm{Au})$ \\
\hline Workpiece dimension & $\begin{array}{l}\text { Indent: } 120 a_{A u} \times 120 a_{A u} \times 120 a_{A u} \\
\text { Scratch: } 160 a_{A u} \times 320 a_{A u} \times 40 a_{A u}\end{array}$ \\
\hline Crystal orientation & Au: $(100),(110),(111)$ \\
\hline
\end{tabular}




\begin{tabular}{|l|l|}
\hline $\begin{array}{l}\text { Number of atoms in the } \\
\text { workpiece }\end{array}$ & $\begin{array}{l}\text { Indent: 6,912,000 atoms } \\
\text { Scratch: 8,192,000 atoms }\end{array}$ \\
\hline Indenter tip material & Diamond \\
\hline Indenter type & Three sided pyramid \\
\hline Indentation depth & $1-7 \mathrm{~nm}$ \\
\hline $\begin{array}{l}\text { Nanoindentation tip } \\
\text { speed }\end{array}$ & $1,10 \mathrm{~m} / \mathrm{s}$ \\
\hline Bulk temperature & $293 \mathrm{~K}$ \\
\hline Time steps & $1 \mathrm{fs}\left(10^{-15} \mathrm{~s}\right)$ \\
\hline
\end{tabular}

\section{EXPERIMENTAL SETUP}

A Veeco Bioscope AFM was used to conduct actual indent and scratch at the nanoscale, and provides data for evaluation of the MD simulation predictions. The AFM provides resolution on the nanometer (lateral) and angstrom (vertical) scales. A diamond probe (Bruker DNISP indentation probe) with a spring constant of $250 \mathrm{~N} / \mathrm{m}$ was used in the experiments. The indenter tips have three-sided pyramid shapes. Nanoindentation is made by forcing the tip into the workpiece until the required cantilever deflection is reached. The tip is then lifted to its initial position above the workpiece. Nanoindentation can be made at various forces and rates, using the deflection of the cantilever as a measure of the indentation force. The indentation force, $F$, is directly proportional to the deflection of the cantilever can be calculated from the well-known Hooke's law:

$$
F=k x
$$

where $k$ is the cantilever stiffness or spring constant in $\mathrm{N} / \mathrm{m}$ and $x$ is the deflection of the cantilever. Nanoscratching is performed by forcing the tip into the workpiece until the required cantilever deflection is reached. The tip is then moved horizontally for a specified length and then lifted to its initial position above the workpiece. The nanoindentation and nanoscratching experiments were conducted at various applied forces and tip velocities.

\section{RESULTS AND DISCUSSION}

MD simulation results of AFM nanoindentation and nanoscratching are presented in this section. All MD simulation snapshots are visualized by Atomeye [67]. The different colors shown in the following figures represent coordination number, which is a measure of how many nearest neighbors exist for a particular atom. For example, atoms in perfect fcc crystals have 12 nearest neighbors and their atomic coordination number is accordingly 12. Atoms with coordination numbers that are not 12 usually represent the location of defects and vacancies. The purpose of using this coordination number coloring is to clearly see the defects and dislocations of atoms.

In nanoindentation process, the indenter tip moves vertically into the surface of substrate. The atoms in the substrate are compressed beneath the tip and the deformation can be seen in the vicinity of the tip. The material apart from the tip seems to effect very little by the motion of the tip. MD simulation snapshots of nanoindentation are shown in Figure 2. The figure shows the initial stage of indenter tip and workpiece material in nanoindentation followed by the movement of the tip into the workpiece material at various time intervals. At the surrounding of contact surface between the indenter tip and the workpiece, a material pile-up is observed. Figure 3 shows top and cross-sectional views of MD simulation snapshots of nanoindentation. The tip is located at the maximum indentation depth at the time of 55 ps (Figure 3 (a)), while the tip is moved to its 
initial point at the time of 115 ps (Figure 3(b)). The elastic deformation on the top surface of the gold workpiece undergoes elastic recovery after the tool tip was moved upward from the workpiece. It can be seen from Figure 3 that some deformation on the surface disappeared after the tool tip was moved up. Moreover, the depth of indentation mark and subsurface deformation decrease after the tip was removed from the workpiece.

Figure 4 shows the MD simulation snapshots of nano-scratching with the scratching depth of 5 $\mathrm{nm}$. The crystal orientation of workpiece material is $\mathrm{Au}(100)$ and the direction of scratching is [100]. The scratching length is $30 \mathrm{~nm}$ and the tip speed is $10 \mathrm{~m} / \mathrm{s}$. The atoms in the workpiece are compressed beneath and in front of the tip and assembled to form a small chip. The material pileup can be seen along the resulting groove. Several types of defects, including vacancies and Shockley partial dislocation loops, can be observed during the simulation. The dislocation loops are highly mobile and participate in various interactions among themselves and with other defects. The dislocation loops on the top surface are emitted in front of the tip and generally move out of the computation domain at a side boundary and come inside from the opposite side of boundary, due to the periodic boundary conditions applied to all four side boundaries.

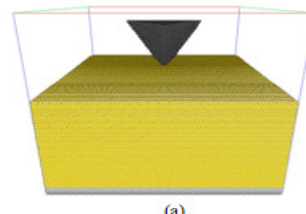

(a)

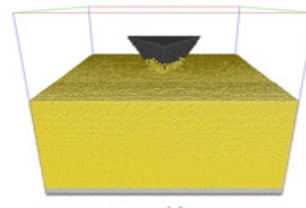

(c)

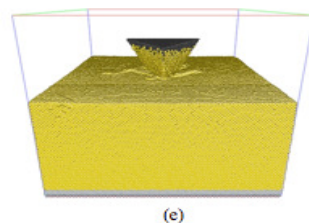

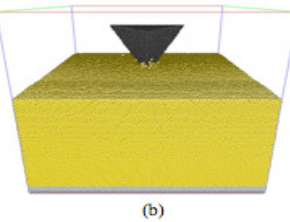

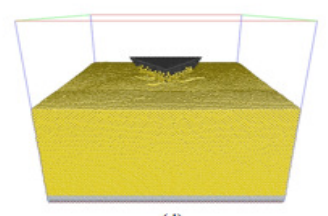

(d)

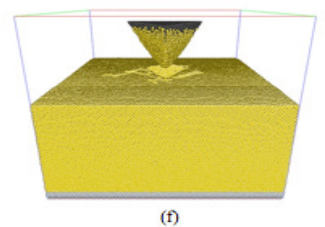

Figure 2. MD simulation snapshots of nano-indentation at various times: (a) 0 ps; (b) $10 \mathrm{ps}$; (c) $30 \mathrm{ps}$; (d) $55 \mathrm{ps}$; (e) $80 \mathrm{ps}$; (f) $115 \mathrm{ps}$
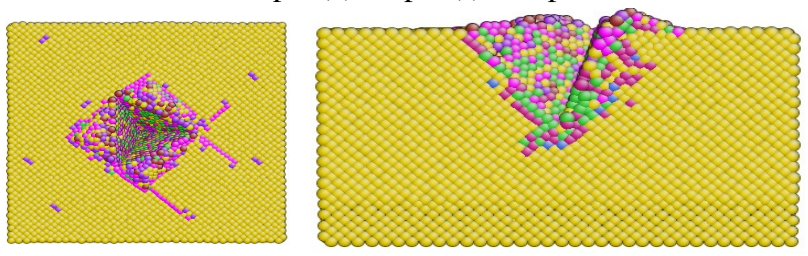

(a)
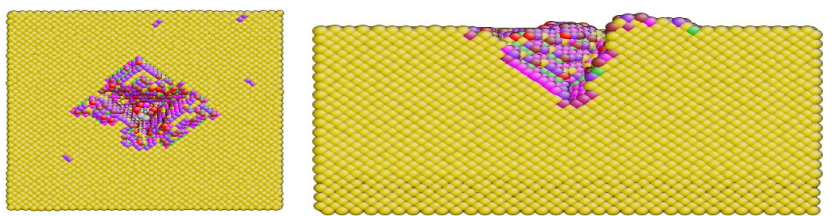

(b)

Figure 3. Top (left) and cross-sectional (right) views of MD simulation snapshots of nano-indentation: (a) time $=55 \mathrm{ps}$; (b) time $=115 \mathrm{ps}$ 

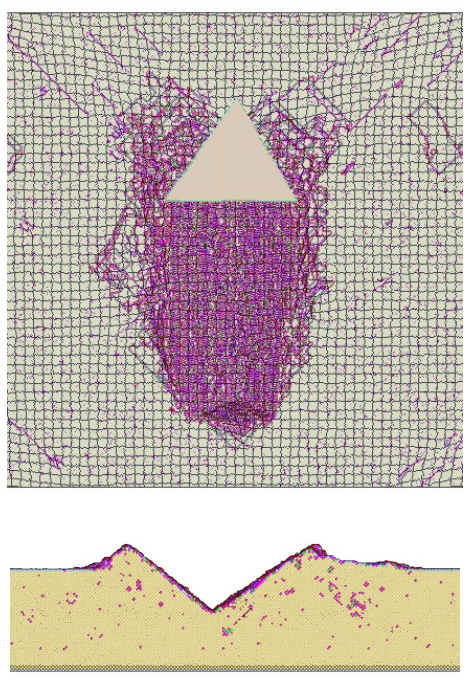

Figure 4. MD simulation snapshots of nano-scratching with a scratching depth of $5 \mathrm{~nm}$.

The effect of scratching depth on material deformation was investigated. MD simulations of nanoscratching were conducted with scratch depths varying from 1 to7 $\mathrm{nm}$. Top and crosssectional views of MD simulation snapshots of nanoscratching at various scratching depths are shown in Figs. 5 - 6, respectively. As the scratching depth increases, the deformation is found to penetrate much deeper from the surface and the height of material pile-up also increases. In addition, more dislocation loops on the top surface can be observed. With increasing depth, the dislocations reach side boundaries sooner and re-enter from the opposite side. Some of these partial dislocations interact with other defects to form more defects on the top surface. This indicates that a larger computation domain is needed.

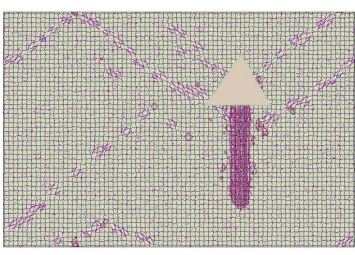

(a)

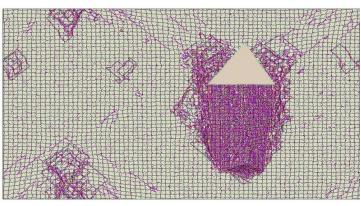

(c)

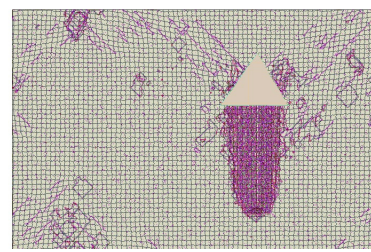

(b)

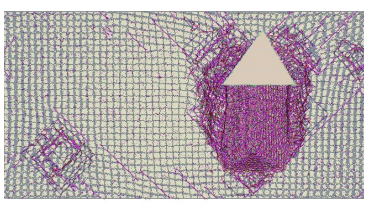

(d)

Figure 5. Top view of MD simulation snapshots of nanoscratching with different depths of scratch: (a) 1 nm; (b) $3 \mathrm{~nm}$; (c) $5 \mathrm{~nm}$; (d) $7 \mathrm{~nm}$. 


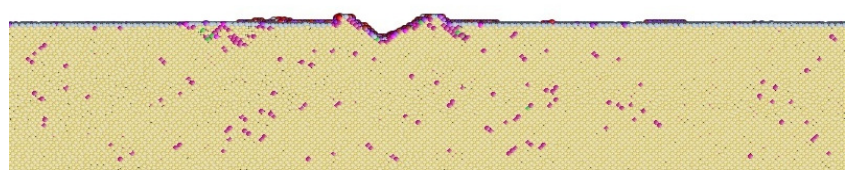

(a)

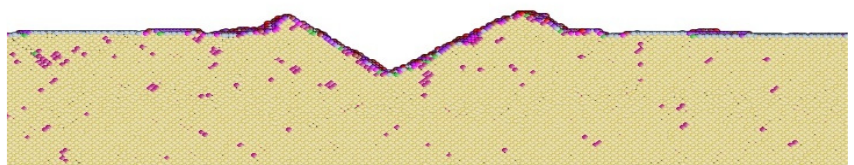

(b)

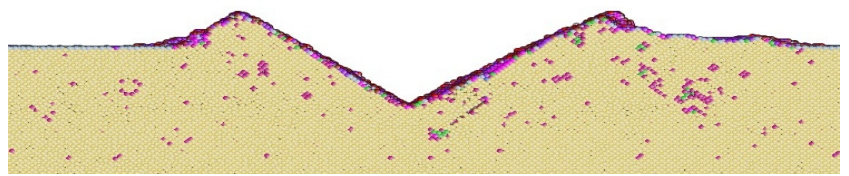

(c)

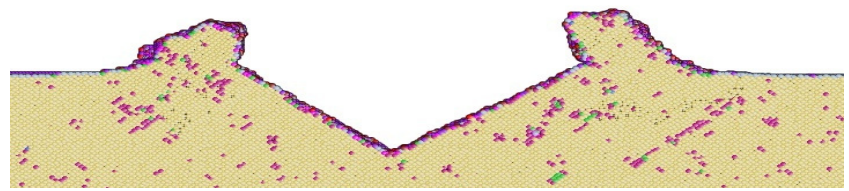

(d)

Figure 6. Cross-sectional views of MD simulation snapshots of nanoscratching with different depths of scratch: (a) $1 \mathrm{~nm}$; (b) $3 \mathrm{~nm}$; (c) $5 \mathrm{~nm}$; (d) $7 \mathrm{~nm}$.

The effects of crystal orientation are presented. Here, the MD simulations of nanoscratching were conducted on three different crystal orientations: $\mathrm{Au}(100), \mathrm{Au}(110)$, and $\mathrm{Au}(111)$. The scratching depths are $5 \mathrm{~nm}$ for all the three cases. Figs $7-9$ show cross-sectional (a, b) and top (c, d) views of different crystal orientations. Different pattern of surface and subsurface deformation can be observed for different crystal orientations.

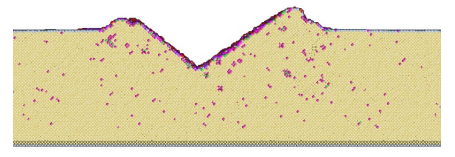

(a)

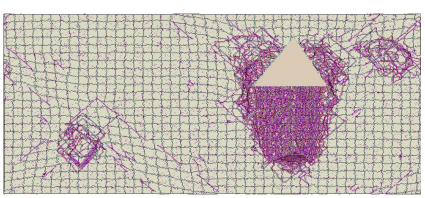

(b)

Figure 7. Cross-sectional (a) and top (b) views of MD simulation snapshots of nanoscratching of $\mathrm{Au}(100)$ 


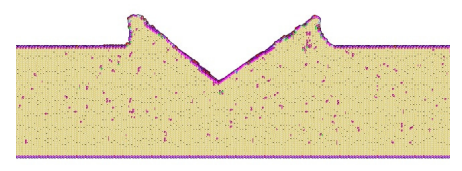

(a)

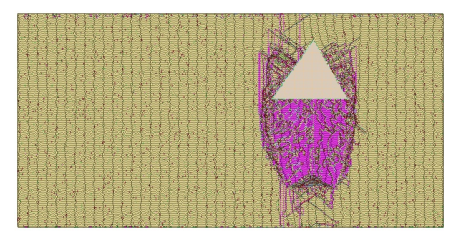

(b)

Figure 8. Cross-sectional (a) and top (b) views of MD simulation snapshots of nanoscratching of $\mathrm{Au}(110)$

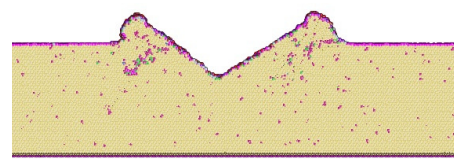

(a)

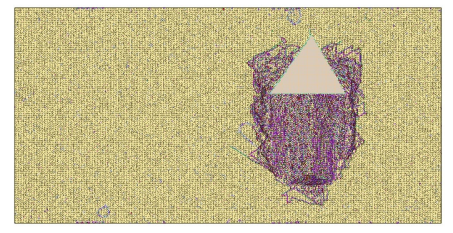

(b)

Figure 9. Cross-sectional (a) and top (b) views of MD simulation snapshots of nanoscratching of $\mathrm{Au}(111)$

Fig. 10 shows AFM image and cross-sectional profile of nanoindentation for different applied forces, increasing from right to left: $65,70,75,80,85 \mu \mathrm{N}$. The AFM experiments were repeated for five times (five rows shown in Fig. 10). The indentation depths increase as the applied forces increase. The variation of indentation forces with depths of indentation at different tool tip speeds is shown in Figure 11. It can be observed that the indentation force increases as the depth of indentation increases. The simulation results were compared with the experimental results. Due to the limitation on computational time, it should be noted that the tool tip speeds used in this MD simulation are a lot higher than those used in the experiment. The typical speed used in the experiment is approximately $5-10 \mu \mathrm{m} / \mathrm{s}$. Therefore, the effect of tool tip speed on the indentation force is also investigated in this paper. It can be observed from Figure 11 that the indentation force increases as the tool tip speed decreases. Since the tip speed plays an important role on the indentation force, the quantitative values of the indentation force obtained from MD simulation are not comparable to the experimental results. However, the increasing trends of indentation force are the same for both simulation and experimental results. AFM experiments of nanoindentation were also carried out to investigate the effect of the tip speed. Fig. 12 shows the experimental results of AFM nanoindentation for different applied forces and different tip speeds. The tip speeds were increased from 1 to $10 \mu \mathrm{m} / \mathrm{s}$ from top to bottom rows. Fig. 12 (b) shows the cross-sectional profile of AFM nanoindentation. The blue line represents the cross-sectional profile for the tip speed of $1 \mu \mathrm{m} / \mathrm{s}$. The red line represents the cross-sectional profile for the tip speed of $10 \mu \mathrm{m} / \mathrm{s}$. It can be seen from Fig. 12 (b) that the indentation depth increases as the tip speed increases. 


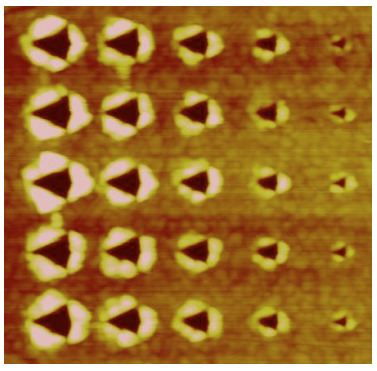

(a)

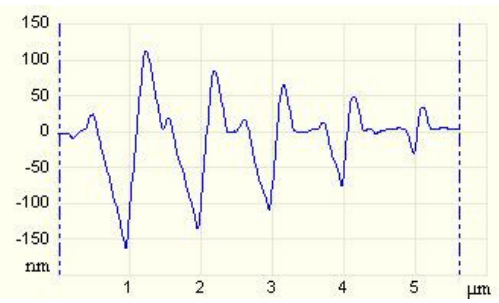

(b)

Figure 10. (a) AFM image of nanoindentation for different applied forces (increasing from right to left); (b) cross-sectional profile of AFM image

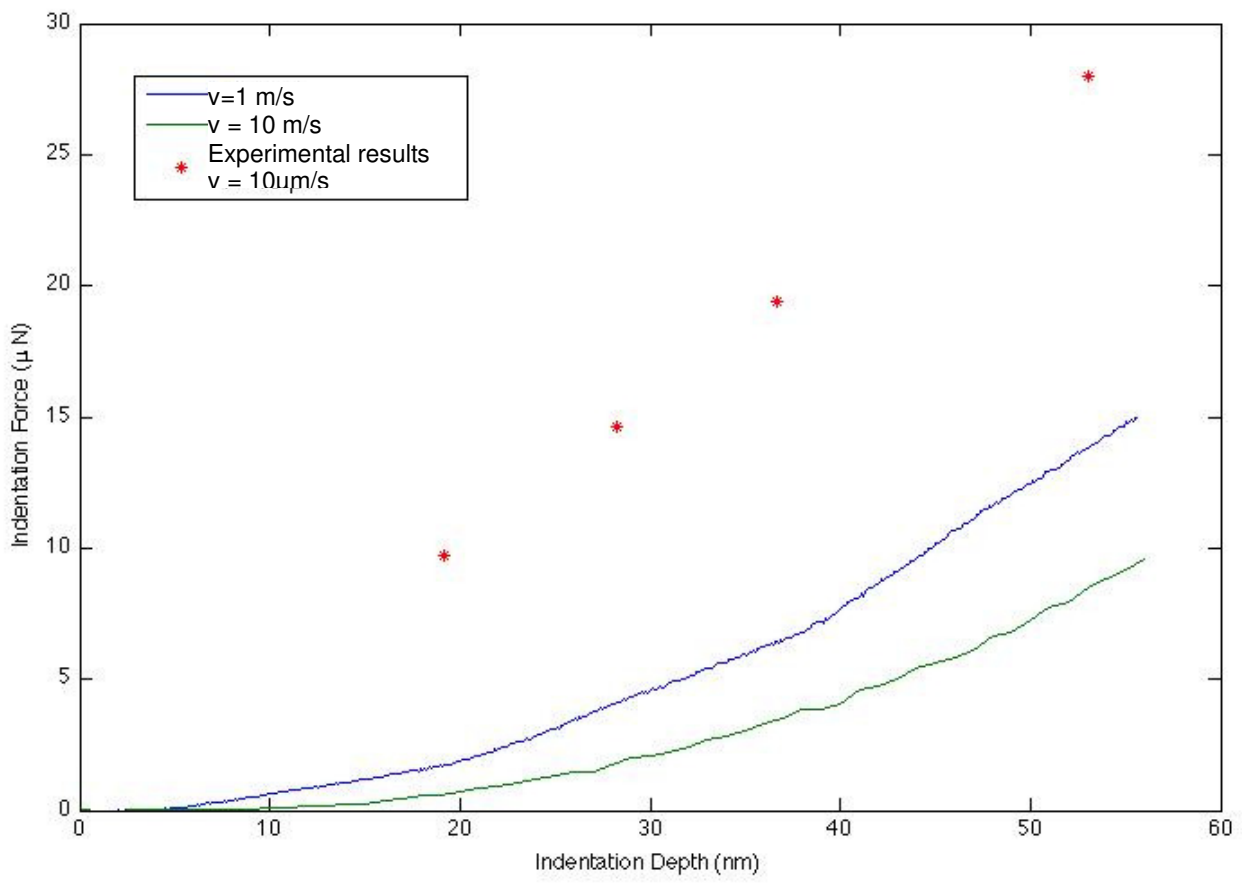

Figure 11. Variation in indentation forces with depths of indentation. 


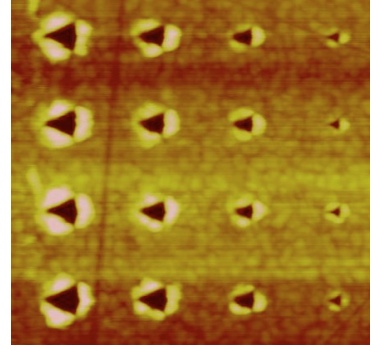

(a)

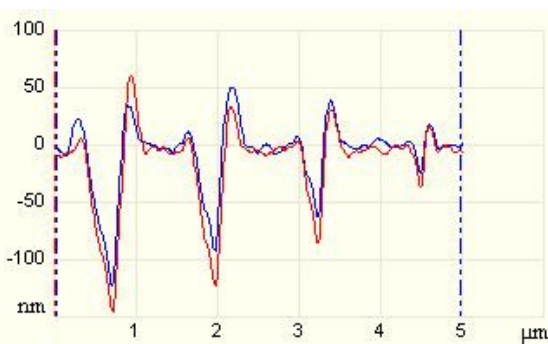

(b)

Figure 12. (a) AFM image of nanoindentation for different applied forces (increasing from right to left) and tip speeds (increasing from top to bottom); (b) cross-sectional profile of AFM image

Figure 13 shows the AFM experimental results of nanoscratching with five different depths, namely 20,30,40, 50, $60 \mathrm{~nm}$ (increasing from right to left). As can be seen from figure 13(b), the surface roughness varies between 0 to $20 \mathrm{~nm}$ across the gold substrate. In order to obtain a meaningful result, the depths of scratch used in the experiments must be higher than $20 \mathrm{~nm}$ which are ten times the depths used in the MD simulation. For this reason, the quantitative values, i.e. forces, obtained from MD simulation are not comparable to the experimental results; only the qualitative values are discussed here. The height of the material pile-up along the scratch groove is found to increase as the depth of cut increases in both MD simulation and AFM experiments. However, the material pile-up on the left side is observed to be higher than the right side which is different from the MD simulation results. One possible explanation for the discrepancy may be that the x-rotation of the AFM probe used in the experiment was set to 12 degree as recommended by manufacturer. During the nanoscratching process, the pile-up material in front of the tip increases, but not enough to form chip. Thus, no chip formation is detected in both MD simulation and experiments for the observed depths of scratch.

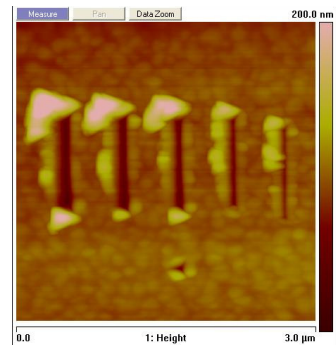

(a)

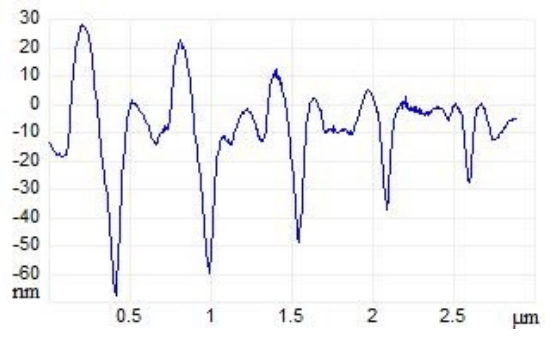

(b)

Figure 13. AFM experimental results of nano-scratching with five scratch depths of 20,30, 40, 50, $60 \mathrm{~nm}$ increasing from right to left: (a) AFM image of nanoscratching (b) cross-sectional profile

Figure 14 shows load-displacement curve for the case of gold material and diamond indenter tip. As the indentation depth of the diamond tip continues to increase, the load curve continues to go up and until its reaches a maximum depth. After reaching the specified maximum depth, the tip begins to unload and return to it original position. The slope of the unloading curve at the maximum load is determined and used in the calculation of hardness and Young's modulus in Eq. (8) and (10), respectively. Two different methods were used to calculate the contact area. In the first method, the contact area was calculated from the geometry of the indenter as a function of the contact depth, $h_{c}$. In the second method, the contact area was estimated from the location of displaced atoms at the interface between tip and sample. The material properties of diamond indenter used in the calculation are $E_{i}=1140 \mathrm{GPa}$ and $v_{i}=0.07$. Table 3 shows the values of Young's modulus and hardness of gold obtained from the calculations. 


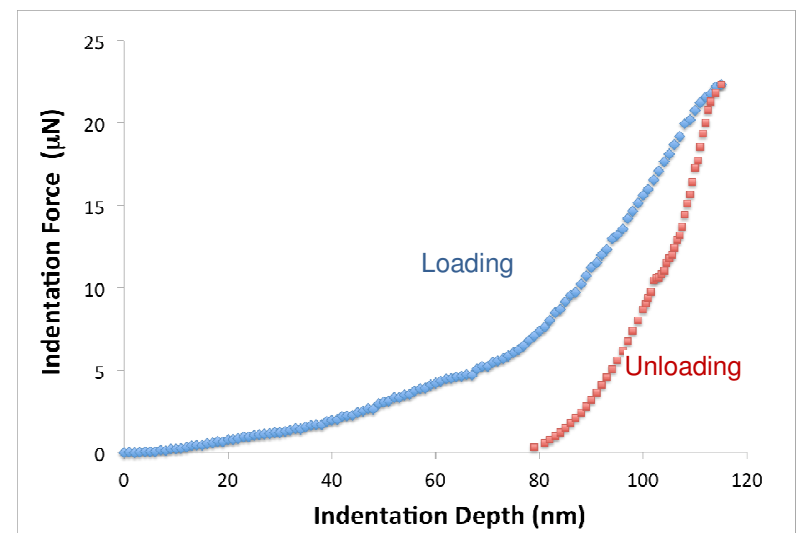

Figure 14. Load-displacement curves for the case of gold material and diamond indenter tip.

Table 3. Young's modulus and hardness of materials at nanoscale

\begin{tabular}{|c|c|c|}
\hline $\begin{array}{c}\text { Young's modulus } \\
(\mathrm{GPa})\end{array}$ & $\begin{array}{c}\text { Hardness } \\
(\mathrm{GPa})\end{array}$ & $\begin{array}{c}\text { Hardness } \\
(\mathrm{GPa})\end{array}$ \\
$\begin{array}{c}\text { Method 1 } \\
\text { Method 2 }\end{array}$ \\
\hline 235 & 113.56 & 98.13 \\
\hline
\end{tabular}

Comparing to the macroscale properties, the Young's modulus of gold is approximately 57-120 $\mathrm{GPa}$; while it was found to be $235 \mathrm{GPa}$ from our calculation. The values of hardness obtained from method 1 in our calculation is slightly larger than those obtained from method 2. Both Young's modulus and hardness in our analysis were about two to three times larger than those at the macroscale. This discrepancy is a result of the scale differences. Bulk material typically has constant material properties regardless of its size, but size-dependent properties are often observed at the nanoscale. Nanoscale material has a high surface area and a large fraction of the atoms are on its surface. This can give rise to size effects in material properties at the nanoscale. Moreover, the defect of the material such as grain boundaries and dislocations was different at different scales. In addition, an assumption of perfect defect-free single-crystal material was applied in the MD simulation; while, in general, materials at macroscale were poly-crystalline and contained several types of defects.

\section{CONCLUSIONS}

MD simulations of AFM-based nanoindentation and nanoscratching were conducted to investigate the effect of indentation and scratching depth, crystal orientation and tip speed. Material properties at the nanoscale were also extracted and compared with macroscopic properties. Several types of defects, including vacancies and Shockley partial dislocation loops, could be observed during the simulation. With increasing depth of scratch, the dislocations reach side boundaries sooner and re-enter from the opposite side. Some of these partial dislocations interact with other defects to form more defects on the top surface. Due to the periodic boundary condition applied to all the four side boundaries, the simulation domain should be large enough to avoid the re-entering of dislocations. For different crystal orientations, different pattern of surface 
and subsurface deformation can be observed. The effect of indentation depths and tip speeds was investigated and found that indentation force increases as depth of indentation and tip speed increase. Material properties, e.g. Young's modulus and hardness, of the materials at the nanoscale are different from those at the macroscale. Hence, due to different material properties between nano- and macro-scale, materials at nanoscale are typically considered new types of material. As can be seen from the presented results, these machining parameters affected the final nano-geometry in AFM-based nanomachining. The findings from this work can be applied to the fabrication of nanochannels/nano-fluidic devices. However, a more extensive experimental study is necessary to better validate the computational models. This will be reported in our future work.

\section{ACKNOWLEDGEMENTS}

This research was supported in part by Lilly Endowment, Inc., through its support for the Indiana University Pervasive Technology Institute, and in part by the Indiana METACyt Initiative. The Indiana METACyt Initiative at IU is also supported in part by Lilly Endowment, Inc.

\section{REFERENCES}

[1] A.P. Malshe, K.P. Rajurkar, K.R. Virwani, C.R. Taylor, D.L. Bourell, G. Levy, M.M. Sundaram, J.A. McGeough, V. Kalyanasundaram and A.N. Samant, "Tip-based nanomanufacturing by electrical, chemical, mechanical and thermal processes," CIRP Annals - Manufacturing Technology, Vol. 59, pp. 628-651, 2010.

[2] A. C. Fischer-Cripps, Nanoindentation, Springer, New York, 2002.

[3] A. N. Shipway, E. Katz and I Willner, "Nanoparticle Arrays on Surfaces for Electronic, Optical, and sensor applications," ChemPhysChem, Vol. 1(1), pp. 18-52, 2000.

[4] M. Liu, N. A. Amro, C. S. Chow and G-Y Liu, "Production of Nanostructures of DNA on Surfaces," Nano Letters, Vol. 2(8), pp. 863-867, 2002.

[5] P. Vettiger, M. Despont, U. Drechsler, U. Dürig, W. Häberle, M. I. Lutwyche, H. Rothuizen, R. Stutz, R. Widmer and G. K. Binnig, "The 'millipede' - more than one thousand tips for future AFM data storage," IBM Journal of Research and Development, Vol. 44(3), pp. 323-340, 2000.

[6] C. R. Taylor, E. A. Stach, G. Salamo and A. P. Malshe, "Nanoscale dislocation Patterning by Ultralow Load Indentation," Applied Physics Letters, Vol. 87(7), 073108, 2005.

[7] G. F. Zheng, F. Patolsky, Y. Cui, W. U. Wang and C. M. Lieber, "Multiplexed electrical detection of cancer markers with nanowire sensor arrays," Nature Biotechnology, Vol. 23(10), pp. 1294-1301, 2005.

[8] X. Li, H. Gao, C. J. Murphy and K. K. Caswell, "Nanoindentation of silver nanowires," Nano Letters, Vol. 3, pp.1495-1498, 2003.

[9] X. Li, P. Nardi, C. W. Baek, J. M. Kim and Y. K. Kim, "Direct nanomechanical machining of gold nanowires using a nanoindenter and an atomic force microscope," Journal of Micromechanics and Microengineering, Vol. 15, pp. 551-556, 2005.

[10] Y. D. Yan, T. Sun, X. S. Zhao, Z. J. Hu and S. Dong, "Fabrication of microstructures on the surface of a micro/hollow target ball by AFM," Journal of Micromechanics and Microengineering, Vol. 18, 035002, 2008.

[11] Y. J. Chen, J. H. Hsu and H. N. Lin, "Fabrication of metal nanowires by atomic force microscopy nanoscratching and lift-off process," Nanotechnology, Vol. 16, pp. 1112-1115, 2005.

[12] Y. T. Mao, K. C. Kuo, C. E. Tseng, J. Y. Huang, Y. C. Lai, J. Y. Yen, C. K. Lee and W. L. Chuang, "Research on three dimensional machining effects using atomic force microscope," Review of Scientific Instruments, Vol. 80, 065105, 2009.

[13] T. Fang, C. Weng and J. Chang, "Machining characterization of nano-lithography process using atomic force microscopy," Nanotechnology, Vol. 11, pp. 181-187, 2000.

[14] S. Diegoli, C. A. E. Hamlett, S. J. Leigh, P. M. Mendes and J. A. Preece, "Engineering nanostructures at surfaces using nanolithography," Proceedings of the Institution of Mechanical Engineers, Part G: Journal of Aerospace Engineering, Vol. 221(4), pp. 589-629, 2007.

[15] T. Sun, Y. D. Yan, J. F. Xia, S. Dong, Y. C. Liang and K. Cheng, "Research on micro machining using AFM diamond tip," Key Engineering Materials, Vols. 259-260, pp. 577-581, 2004. 
[16] J. C. Huang, C. L. Li, and J. W. Lee, "The Study of Nanoscratch and Nanomachining on Hard Multilayer Thin Films Using Atomic Force Microscope," Scanning, Vol. 34, pp. 51-59, 2012.

[17] Y. Yan, T. Sun, Y. Liang and S. Dong, "Investigation on AFM-based micro/nano-CNC machining system,” International Journal of Machine Tools and Manufacture, Vol. 47, pp. 1651-1659, 2007.

[18] F. Zhang, H. Zhang, Y. Yan and J. Wang, " Research on nano-scale material removal process using atomic force microscopy," Key Engineering Materials, Vols. 359-360, pp. 269-273, 2008.

[19] H. Zhang, J. Kuai and F. Zhang, "Minimum thickness of cut in nanomachining using atomic force microscopy," 2010 International Conference on E-Product E-Service and E-Entertainment (ICEEE), Henan, China, November 7-9, 2010.

[20] A. A. Tseng, J. Shirakashi, S. Nishimura, K. Miyashita and Z. Li, "Nanomachining of permalloy for fabricating nanoscale ferromagnetic structures using atomic force microscopy," Journal of Nanoscience and Nanotechnology, Vol. 10, pp. 456-466, 2010.

[21] Z. Q. Wang, N. D. Jiao, S. Tung and Z. L. Dong, "Atomic force microscopy-based repeated machining theory for nanochannels on silicon oxide surfaces," Applied Surface Science, Vol. 257, pp. 3627-3631, 2011.

[22] W. C. D. Cheong and L. C. Zhang, "Molecular dynamics simulation of phase transformations in silicon monocrystals due to nano-indentation," Nanotechnology, Vol. 11, pp. 173-180, 2000.

[23] R. Komanduri, N. Chandrasekaran and L.M. Raff, "MD simulation of indentation and scratching of single crystal aluminum," Wear, Vol. 240, pp. 113-143, 2000.

[24] D. Christopher, R. Smith and A. Richter, "Atomistic modelling of nanoindentation in iron and silver," Nanotechnology, Vol. 12, pp. 372-383, 2001.

[25] T. Fang, C. Weng and J. Chang, "Molecular dynamics analysis of temperature effects on nanoindentation measurement," Material Science and Engineering, Vol. A357, pp. 7-12, 2003.

[26] X. M. Liu, Z. L. Liu and Y G Wei, "Nanoscale Friction Behavior of the Ni-film/substrate system under scratching using MD simulation," Tribology Letters Vol. 46, pp. 167-178, 2012.

[27] A. Gannepalli and S. K. Mallapragada, "Molecular dynamics studies of plastic deformation during silicon nanoindentation," Nanotechnology, Vol. 12, pp. 250-257, 2001.

[28] I. Salehinia, S.K. Lawrence and D.F. Bahr, "The effect of crystal orientation on the stochastic behavior of dislocation nucleation and multiplication during nanoindentation," Acta Materialia, Vol. 61, pp. 1421-1431, 2013.

[29] C. F. Sanz-Navarro, S. D. Kenny and R. Smith, "Atomistic simulations of structural transformations of silicon surfaces under nanoindentation," Nanotechnology, Vol. 15, pp. 692-697, 2004.

[30] T. Akabane, Y. Sasajima and J. Onuki, "Computer simulation of silicon nanoscratch test," Materials Transactions, Vol. 47, pp. 1090-1097, 2006.

[31] H. Okabe, T. Tsumura, J. Shimizu, L. Zhou and H. Eda, "Experimental and Simulation Research on Influence of Temperature on Nano-Scratching Process of Silicon Wafer," Key Engineering Materials, Vol. 329, pp. 379-384, 2007.

[32] T. Fang, W. Chang and C. Weng, "Nanoindentation and nanomachining characteristics of gold and platinum thin films," Materials Science and Engineering A, Vol. 430, pp. 332-340, 2006.

[33] T. Fang and C. Weng, "Three-dimensional molecular dynamics analysis of processing using a pin tool on the atomic scale," Nanotechnology, Vol. 11, pp. 148-153, 2000.

[34] T. Fang, C. Weng, and J. Chang, "Molecular dynamics simulation of nano-lithography process using atomic force microscopy,” Surface Science, Vol. 501, pp. 138-147, 2002.

[35] Y. Yan, T. Sun, S. Dong and Y. Liang, "Study on effects of the feed on AFM-based nano-scratching process using MD simulation," Computational Materials Science, Vol. 40, pp. 1-5, 2007.

[36] H. Yu, J. B. Adams and L. G. Hector Jr, "Molecular dynamics simulation of high-speed nanoindentation," Modeling and Simulation in Materials Science and Engineering, Vol. 10, pp. 319$329,2002$.

[37] S. Jun, Y. Lee, S. Kim and S. Im, "Large-scale molecular dynamics simulations of Al(111) nanoscratching," Nanotechnology, Vol. 15, pp. 1169-1174, 2004.

[38] Y. Lee, J. Park, S. Kim, S. Jun and S. Im, "Atomistic simulations of incipient plasticity under $\mathrm{Al}(111)$ nanoindentation," Mechanics of Materials, Vol. 37, pp. 1035-1048, 2005.

[39] D. Mulliah, D. Christopher, S. D. Kenny and R. Smith, "Nanoscratching of silver (100) with a diamond tip," Nuclear Instruments and Methods in Physics Research B, Vol. 202, pp. 294-299, 2003.

[40] D. Mulliah, S. D. Kenny, R. Smith and C. F. Sanz-Navarro, "Molecular dynamics simulations of nanoscratching of silver (100)," Nanotechnology, Vol. 15, pp. 243-249, 2004.

[41] R. Smith, D. Cristopher and S. D. Kenny, "Defect generation and pileup of atoms during nanoindentation of Fe single crystals," Physical Review B, Vol. 67, 245405, 2003. 
[42] C. Lu, Y. Gao, G. Y. Deng, G. Michal, N. N. Huynh, X. H. Liu and A. K. Tieu, “Atomic-scale anisotropy of nanoscratch behavior of single crystal iron," Wear, Vol. 267, pp. 1961-1966, 2009.

[43] Z. Lin, J. Huang and Y. Jeng, "3D nano-scale cutting model for nickel material," Journal of Materials Processing Technology, Vol. 192-193, pp. 27-36, 2007.

[44] Y. Gao, C. Lu, N. N. Huynh, G. Michal, H. T. Zhu and A. K. Tieu, "Molecular dynamics simulation of effect of indenter shape on nanoscratch of Ni," Wear, Vol. 267, pp. 1998-2002, 2009.

[45] I. Gheewala, R. Smith and S. D. Kenny, "Nanoindentation and nanoscratching of rutile and anatase TiO2 studied using molecular dynamics simulations," Journal of Physics: Condensed Matter, Vol. 20, 354010, 2008.

[46] Y. Liang, J. Chen, M. Chen, D. Song and Q Bai, “Three-dimensional molecular dynamics simulation of nanostructure for reciprocating nanomachining process," Journal of Vacuum Science and Technology B, Vol. 27, pp. 1536-1542, 2009.

[47] J. Chen, Y. Liang, Q. Bai, Y. Tang and M. Chen, "Mechanism of material removal and the generation of defects by MD analysis in three-dimensional simulation in abrasive processes," Key Engineering Materials, Vol. 359-360, pp. 6-10, 2008.

[48] Z. Lin and J. Huang, "A study of the estimation method of the cutting force for a conical tool under nanoscale depth of cut by molecular dynamics," Nanotechnology, Vol. 19, 11570, 2008.

[49] D. Mulliah, S. D. Kenny, E. McGee, R. Smith, A. Richter and B. Wolf, "Atomistic modeling of ploughing friction in silver, iron and silicon," Nanotechnology, Vol. 17, pp. 1807-1818, 2006.

[50] R. Promyoo, H. El-Mounayri, and A. Martini, "AFM-Based Nanomachining for Nano-fabrication Processes: MD Simulation and AFM Experimental Verification," ASME International Manufacturing Science \& Engineering Conference, Erie, PA, October 2010.

[51] R. Promyoo, H. El-Mounayri, K. Varahramyan, and A. Martini, "Molecular Dynamics Simulation of AFM-Based Nanomachining Processes," ASME International Manufacturing Science \& Engineering Conference, Corvallis, OR, June 13 - 17, 2011.

[52] R. Promyoo, H. El-Mounayri, and K. Varahramyan, "AFM-Based Manufacturing for Nanofabrication Processes," TSME International Conference on Mechanical Engineering, Krabi, Thailand, October 2011.

[53] R. Promyoo, H. El-Mounayri, and K. Varahramyan, "AFM-Based Nanoindentation Process: A Comparative Study," ASME International Manufacturing Science \& Engineering Conference, Norte Dame, IN, June 4-8, 2012.

[54] R. Promyoo, H. El-Mounayri, K. Varahramyan, and V. Kumar, "AFM-Based Nanofabrication: Modeling, Simulation, and Experimental Verification," ASME International Manufacturing Science \& Engineering Conference, Madison, WI, June 10 - 14, 2013.

[55] R. Promyoo, H. El-Mounayri, and K. Varahramyan, "AFM-based nanoindentation using a 3D molecular dynamics simulation model," Journal of Materials Science and Engineering A, Vol. 3(6), pp. 369-381, 2013.

[56] B. J. Alder and T. E. Wainwright, 1959, "Studies in molecular dynamics. I. General method," Journal of Chemical Physics, Vol. 31, pp. 459-466, 1959.

[57] B. J. Alder and T. E. Wainwright, "Studies in molecular dynamics. II. Behavior of a small number of elastic spheres," Journal of Chemical Physics, Vol. 33, pp. 1439-1451, 1960.

[58] R. Komanduri and L. M. Raff, "A review on the molecular dynamics simulation of machining at the atomic scale," Proceedings Institution of Mechanical Engineers, Vol. 215 (B), pp. 1639-1672, 2001.

[59] S. J. Plimpton, S. J., "Fast parallel algorithms for short-range molecular dynamics," Journal of Computational Physics, Vol. 117, pp. 1-19, 1995.

[60] S. J. Plimpton, R. Pollock and M. Stevens, "Particle-mesh Ewald and rRESPA for parallel molecular dynamics simulations," Proc of the Eighth SIAM Conference on Parallel Processing for Scientific Computing, Minneapolis, MN.

[61] P. Walsh, R. K. Kalia, A. Nakano and P. Vashishta, "Amorphization and anisotropic fracture dynamics during nanoindentation of silicon nitride: A multimillion atom molecular dynamics study," Applied Physics Letters, Vol.77, pp.4332-4334, 2000.

[62] M. S. Daw and M. I. Baskes, "Embedded-atom method: Derivation and application to impurities, surfaces, and other defects in metals," Physical Review B, Vol. 29, pp. 6443-6453, 1984.

[63] P. M. Morse, "Diatomic molecules according to the wave mechanics II vibrational levels," Physical Review, Vol. 34, pp. 57-64, 1929.

[64] I. M. Torrens, Interatomic potentials, Academic, New York, 1972. 
[65] W.C. Oliver, G.M. Pharr, "Measurement of hardness and elastic modulus by instrumented indentation: Advances in understanding and refinements to methodology," Journal of Material Research, Vol. 19, pp. 3-20, 2004.

[66] http://rt.uits.iu.edu/bigred2/index.php

[67] J. Li, "AtomEye: an efficient atomistic configuration viewer," Modeling and Simulation in Materials Science and Engineering, Vol. 11, pp. 173-177, 2003. 